\title{
Large polycyclic aromatic hydrocarbons for application in donor-acceptor photovoltaics
}

\author{
Holger Christian Hesse', Christoph Schaffer', Christian Hundschell', Akimitsu Narita', Xinliang Feng ${ }^{2}$ \\ Klaus Müllen², Bert Nickel', and Lukas Schmidt-Mende ${ }^{*, 1,3}$ \\ ${ }^{1}$ Department of Physics and Center for Nanoscience (CeNS), Ludwigs Maximilians University Munich, Amalienstr. 54, \\ 80799 Munich, Germany \\ ${ }^{2}$ Max Planck Institute for Polymer Research, Ackermannweg 10, 55128 Mainz, Germany \\ ${ }^{3}$ Department of Physics, University of Konstanz, Universitätsstrasse 10, 78457 Konstance, Germany
}

Keywords exciton diffusion, graphene, organic photovoltaics, photoluminescence quenching

${ }^{*}$ Corresponding author: e-mail Lukas.Schmidt-Mende@uni-konstanz.de, Phone: +49 753188 5409, Fax: +49 7531884788 ;

Web: hybrid-nanostructures.physik.lmu.de and cms.uni-konstanz.de/physik/schmidt-mende/

We present the photovoltaic application of a donor-acceptor system consisting of very large polycyclic aromatic hydrocarbons. Using vacuum sublimated hexa-peri-hexabenzocoronenes we evidence long exciton diffusion lengths of approximately $25 \mathrm{~nm}$. In conjunction with the heaxfluorinated analogue for the first time a photovoltaic device using nanographene as active material for both donor and acceptor compounds was fabricated. The bi-layered device exhibits a remarkably high open circuit voltage of $1.39 \mathrm{~V}$. Light absorption of the photoactive materials used here is strictly confined to wavelengths below $500 \mathrm{~nm}$, rendering this approach especially interesting for the application in semi-transparent devices as well as multi-layered and tandem solar cells.

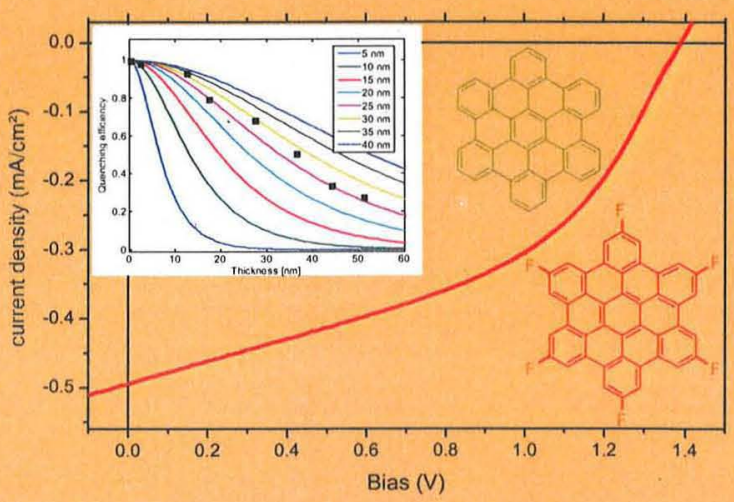

The photovoltaic performance of devices based on vacuum sublimated hexa-peri-hexabenzocoronenes is shown.
1 Introduction Hexa-peri-hexabenzocoronene (HBCs) is an outstanding p-type organic semiconductor material with a core unit consisting of thirteen fused benzene rings. Self-assembled, organized 1D-structures [1-4] and very high charge-carrier mobility values reaching $1.1 \mathrm{~cm}^{2} / \mathrm{Vs}$ have been evidenced in the bulk state for $\mathrm{HBC}$ derivatives $[5,6]$. In addition, as shown in this letter, HBCs can show an exceptionally high exciton diffusion length of around $25 \mathrm{~nm}$.

HBCs have been previously utilized as donor materials in OPV devices showing an external quantum efficiency of around $30 \%$ in combination with perylene-diimide as acceptor material $[7,8]$.

The introduction of electron-deficient groups at residual positions of organic semiconductors may allow to strongly influence their electronic properties: for example, fluoro[9-12] and cyano- [13] groups incorporated to p-type semiconductor materials resulted in the formation of closely related materials with n-type semiconducting character.

Hexafluoro-HBC (HBC-6F) was reported to show n-type properties with HOMO and LUMO levels of -5.9 and 
$-3.2 \mathrm{eV}$, respectively, both of which are $0.5 \mathrm{eV}$ lower than the non-substituted HBC [14]. The band gap was not affected by the fluoro-substitution resulting in an almost unchanged optical absorption of the molecules. However, the interface dipole and the ionization potential are enhanced compared to the non-substituted HBC (HBC) [15].

Organic field-effect transistors assembled using thin films of HBC-6F show an electron field-effect mobility of $1.6 \times 10^{-2} \mathrm{~cm}^{2} / \mathrm{Vs}$ and on/off ratio of $10^{4}[16,17]$. Furthermore, HBC-6F was found to form a face-to-face structure, which is advantageous for the charge-carrier transport [15].

Combining donor and acceptor materials originating from a common core molecule seems appealing for the application in photovoltaic devices: Perfluorinated subphthalocyanine has been used in conjunction with nonsubstituted subphthalocyanine in an organic solar cell showing a high open-circuit voltage $\left(V_{\mathrm{OC}}\right)$ of $0.94 \mathrm{~V}$ and a power conversion efficiency of $0.96 \%$ [18].

Recently the use of non-fullerene acceptor molecules for the application in OPV devices has drawn an increasing attention [19] and their individual advantages and drawbacks have been analyzed. HBC-6F is a new candidate with a planar core shape and a suitable energy level alignment. The HOMO is found to be suitable for exciton separation at the heterojunction and the larger bandgap in $\mathrm{HBC}-6 \mathrm{~F}$ potentially allows an increase in $V_{\mathrm{OC}}$ [20].

We conceived that the combination of non-substituted $\mathrm{HBC}$ and HBC-6F in OPV devices as donor and acceptor materials, respectively, would be desirable for power conversion using high energy photons. It is worth mentioning, that this is the first time only nanographene materials are used as photoactive materials in an OPV device.

2 Results and discussion Figure 1 shows the molecules used in this study. HBC has shown suitable properties for the application in OPV devices, previously [21]. The hexafluorinated analogue instead has not been tested for photovoltaic applications before. Combining the materials with a nanographene core in a donor-acceptor heterojunction renders a novel approach to energy harvesting using thin film devices.

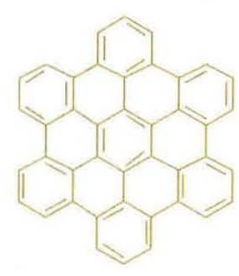

$\mathrm{HBC}$

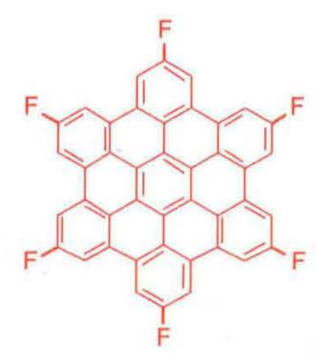

HBC-6F
Figure 1 (online color at: www.pss-a.com) Schematic representation of the molecules used in this study: un-substituted HBC and the hexafluoro-HBC.
2.1 Film growth The growth of organic films of HBC-patent and HBC-6F was analyzed using optical absorption, X-ray, and atomic force microscopy (AFM) imaging techniques.

Figure 2 shows the optical absorption spectra of vacuum sublimated thin films of $\mathrm{HBC}$ and $\mathrm{HBC}-6 \mathrm{~F}$ deposited on quartz glass. A very similar absorption spectrum with an onset at $470 \mathrm{~nm}$ and peak absorption at about $355 \mathrm{~nm}$ is observed for both HBC and HBC-6F films. A slightly lower absorbance is observed for the hexafluorinated derivative, which we attribute to a decreased density of chromophores due to the sterical demand of the fluorine groups increasing the molecular volume.

For the organic materials discussed in this paper, XRD measurements have been performed to analyze the supermolecular organization of the molecules.

It is known from literature, that layer growth and alignment of the molecules may have a great impact on the photo-physical properties of resulting films [22, 23].

For pristine layers of HBC a clear peak at $q=0.53 \pm 0.02$ could be evidenced. This peak corresponds to a net layer distance of $11.9 \pm 0.4 \AA$.

Based on the unit cell shown for the non-substituted HBC [24], a simulation of the (001) diffraction predicts a peak position of $d=11.94 \AA$, in good agreement with the experimentally observed result (schematically shown in the inset of Fig. 3). Instead, for films of HBC-6F grown on silicon nitride and ITO substrates (Fig. 3, black line) no reflections could be resolved with our experimental setup. The absence of crystallization peaks gives rise to the assumption that $\mathrm{HBC}-6 \mathrm{~F}$ grows in an amorphous phase at the film deposition conditions chosen. For bi-layered stacks consisting of $\mathrm{HBC}$ and $\mathrm{HBC}-6 \mathrm{~F}$, again only the peak assigned to the pristine $\mathrm{HBC}$ donor layer is apparent $(11.9 \AA)$.

We conclude that the HBC-6F grows in an amorphous phase on all underlying substrates and conditions investigated. Calculated reflection positions for the non-substituted HBC are well in accordance with the peak position experimentally determined (Fig. 3). Comparing these results with the information given above, we infer that $\mathrm{HBC}$ is

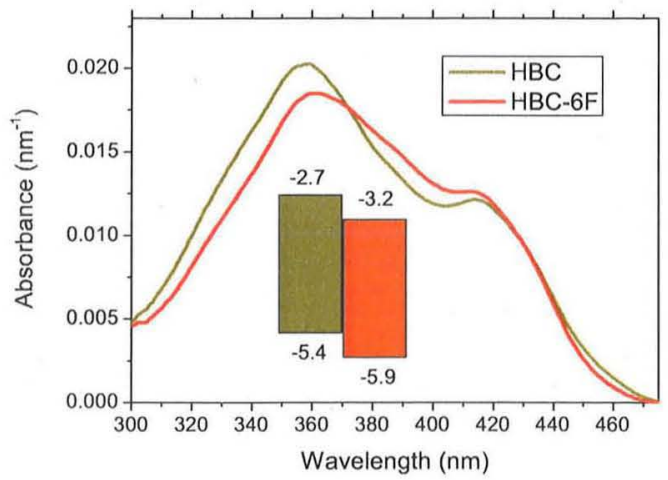

Figure 2 (online color at: www.pss-a.com) Optical absorption and band level alignment of the molecules HBC and HBC-6F. 


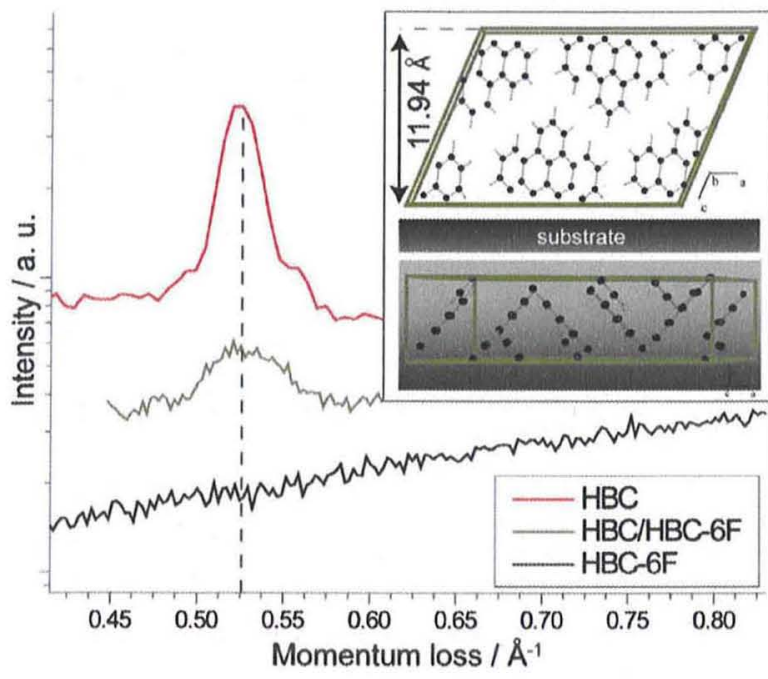

Figure 3 (online color at: www.pss-a.com) XRD spectra of HBC, HBC/HBC- $6 \mathrm{~F}$, and HBC-6F thin films on ITO-covered glass substrates. The first simulated peak position for the donor material HBC is indicated by the dotted line. The inset image shows the unit cell obtained for HBC [24] and its orientation to the substrate (gray) as inferred from the XRD data.

growing with the $a b$-plane as its basal plane and with no indication for developing a thin film phase.

2.2 Exciton diffusion length in unsubstituted HBC The exciton diffusion length of the non-substituted HBC molecules is determined via a photoluminescence quenching study. Similar to the method reported by Markov et al., the quenching yield of the HBC fluorescence upon addition of an acceptor is analyzed [25].

Perylene-diimide is applied onto the smooth HBC donor layers as an effective exciton quencher-this molecule exhibits both absorption and PL emission spectra, which can be well distinguished from the signal obtained for the sublimated donor material $[7,8]$.

Very strong exciton quenching is observed for excited states in the $\mathrm{HBC}$ layer, when diffusing to the interface to the solution deposited n-type semiconductor.

$$
Q=\frac{\left[a^{2} L_{\mathrm{D}}^{2}+a L_{\mathrm{D}} \tanh \left(\frac{L}{L_{\mathrm{D}}}\right) \exp (-a L)-\frac{a^{2} L_{D}^{2}}{\cosh \left(L / L_{\mathrm{D}}\right)}\right]}{\left(1-a^{2} L_{\mathrm{D}}^{2}\right)(1-\exp (-a L))}
$$

As evident from formula (1) the quenching rate $Q$ can be calculated as a function of HBC layer thickness $L$ and the absorption coefficient $a$ of the organic material [25]. $L_{\mathrm{D}}$ refers to the exciton diffusion length characteristic to this layer. As such, the quenching rate $\mathrm{Q}$ can be directly correlated to the exciton diffusion length of the pristine HBC donor layers.

It is worth mentioning, that AFM measurements on the evaporated HBC layers confirm a smooth surface with an

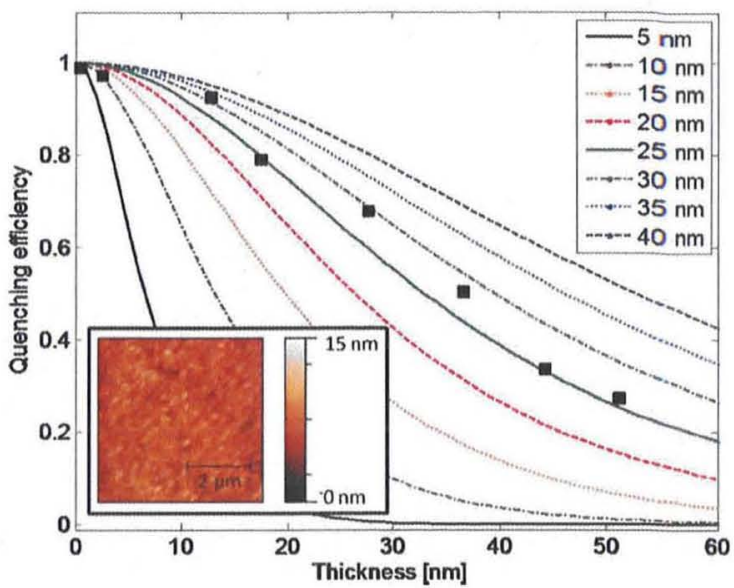

Figure 4 (online color at: www.pss-a.com) Quenching rate analysis for the vacuum processed donor material HBC. Black squares indicate quenching rates obtained depending on the thicknesses of HBC. Colored lines are derived using formula (1) at an $L_{\mathrm{D}}$ as shown in the figure legend. The inset image shows a representative AFM scan of the non-substituted HBC surface.

RMS roughness of $<1 \mathrm{~nm}$ for all samples. An increased surface roughness will lead to an overestimation of the exciton diffusion length when applying formula (1).

The photoluminescence of the thin films was measured at a fixed emission wavelength of $580 \mathrm{~nm}$ and optical excitation in the wavelength region of $300-400 \mathrm{~nm}$ before and after the deposition of PDI. Figure 4 shows the experimental data obtained along with calculated slopes for different values of $L_{\mathrm{D}}$. Best fit to the experimental data is obtained with $L_{\mathrm{D}}=(25 \pm 5) \mathrm{nm}$ for the non-substituted HBC layer. This finding is also in accordance to external quantum efficiency measurements conducted on planar HBC-PDI solar cells with a varying HBC layer thicknesses $\left(d_{\mathrm{HBC}}\right)$ showing peak performance at $d_{\mathrm{HBC}}=25 \mathrm{~nm}$ (data not shown).

An exciton diffusion length of around $25 \mathrm{~nm}$ as shown here renders $\mathrm{HBC}$ very appealing for applications in photovoltaic devices: common solution processed donor polymers frequently used in OPV exhibit significantly lower diffusion lengths of $10 \mathrm{~nm}$ and below [26]. As such, an intimate intermixing of donor and acceptor materials is necessary for efficient exciton separation [27].

Similarly, for most sublimated small molecules an exciton diffusion length in the range of $10-20 \mathrm{~nm}$ has been evidenced in a recent study [28]. The exceptionally high exciton diffusion length found here for the donor material HBC allows the incorporation in bi-layered devices with increased device thickness providing additional photon harvesting.

2.3 Photovoltaic devices HBC and HBC-6F have been used as donor and acceptor materials to fabricate bilayered photovoltaic devices. A device structure composed of ITO/PEDOT:PSS/HBC|HBC-6F/LiF/Al was chosen and 


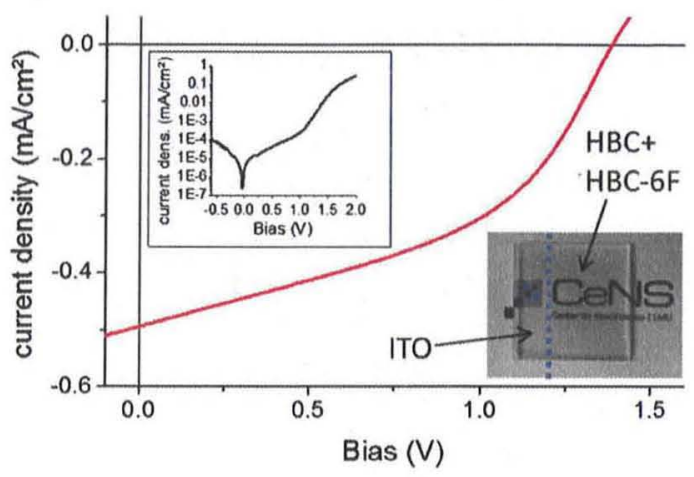

Figure 5 (online color at: www.pss-a.com) Current-voltage characteristics of a bi-layered photovoltaic device with active layer HBC $(30 \mathrm{~nm}) \mathrm{HBC}-6 \mathrm{~F}(20 \mathrm{~nm})$. The dark characteristic of the device is shown in the inset plot. The lower right image shows a photograph of the UV-vis light absorbing organic layers on ITO support.

layers deposited as is further described in the experimental section.

Figure 5 shows a typical current-voltage characteristic obtained for a bi-layered device with the nanographene materials as photoactive compounds.

Under illumination at standard testing conditions $\left(100 \mathrm{~mW} / \mathrm{cm}^{2}, \mathrm{AM} 1.5 \mathrm{~g}\right.$ spectrum) the device shows a $V_{\mathrm{OC}}$ of $1.39 \mathrm{~V}$, a fill factor of 0.45 , and short circuit current density $\left(I_{\mathrm{SC}}\right)$ of $0.50 \mathrm{~mA} / \mathrm{cm}^{2}$, resulting in a power conversion efficiency of around $0.35 \%$.

We believe that the fill factor of the device is mainly limited by the non-crystalline packing and therefore most likely low mobility obtained in the HBC-6F (as discussed in Section 2.1.). Increased current generation might become achievable when the molecular stacking and charge carrier mobility are improved [29]. Significant space charge might built up under working conditions lowering the maximum attainable $V_{\mathrm{OC}}$ and causing a slight "s"-shape of the $I-V$ characteristics near $V_{\mathrm{OC}}$ as observed here. The inset image of Fig. 5 shows the dark current density of the device. A rectification of $2 \times 10^{3}$ is found at a $2 \mathrm{~V}$ bias.

The high open circuit potential obtained for the device seems very appealing for direct use in technical applications [17]. Furthermore, the active layer of the photovoltaic devices presented show an absorption onset at below $500 \mathrm{~nm}$ (Fig. 5, inset photograph). Window-integrated UV-light harvesting might become feasible using the high-energy photon harvesting concept presented here.

Multilayer and tandem device concepts have drawn an increasing attention in the field of OPV $[30,31]$. The UVabsorber combination shown here could be combined with a polymer-fullerene based vis-near-IR absorbing subcell allowing for strongly increased photon harvesting [32, 33].

It is also worth mentioning here that HBCs exhibit an exceptional thermal stability of up to $400^{\circ} \mathrm{C}$, rendering this donor-acceptor combination suitable for new fields of applications where the materials are exposed to harsh environmental conditions. A $6-\mathrm{h}$ annealing treatment at $160^{\circ} \mathrm{C}$ resulted in an almost unchanged photovoltaic performance of the device. At even higher temperatures instead, a performance decrease is induced by strong "s"-shape formation near the $V_{\mathrm{OC}}$ condition, which we attribute to a degradation of the interface and contact materials.

3 Conclusion Processing of hexa-peri-hexabenzocoronene from the vapor phase allows growing thin films with properties desired for the application in photovoltaic devices. A remarkably high exciton diffusion length of $L_{\mathrm{D}} \approx 25 \mathrm{~nm}$ could be demonstrated for the non-substituted disc shaped molecule.

In combination with a fluorinated derivative we have fabricated the first OPV devices using nanographene as both donor and acceptor materials and delivered a proof of principle; bi-layered devices were assembled which allow harvesting UV light and yield a high open circuit voltage of $1.39 \mathrm{~V}$. Further optimization of the system will be necessary to improve the power conversion efficiency. Higher mobility values and a reduced space charge of devices are expected if crystalline growth can be induced in the fluorinated HBC. On the other hand, co-evaporation of the organic materials might allow to increase the exciton harvesting yield of the devices [34]. At controlled conditions an intermixed phase with well aligned but segregated domains of $\mathrm{p}$ - and n-type materials might be formed as motivated by side chain pairs with an incompatible packing [35].

The high $L_{\mathrm{D}}$ value obtained for the unsubstituted HBC also renders the molecule very appealing for the application in interdigitated nano-structured morphology concepts [36]. A pillar spacing in the order of the exciton diffusion length might allow for exhaustive photon harvesting for wavelengths below the absorption onset [37].

\section{Experimental methods}

4.1 Photoluminescence analysis Steady-state PL measurements have been conducted on the same samples with a Horiba Yvon Fluorolog PL spectrometer equipped with a $500 \mathrm{~W}$ halogen lamp.

4.2 UV-vis measurements An Agilent Technologies UV-vis spectrometer (8453) was used for the acquisition of absorption spectra.

4.3 Device preparation ITO substrates are cleaned by immersion in utrasonication baths using a detergent saturated deionized water mixture, acetone, and isopropyl alcohol solution subsequently. $\mathrm{An}_{2}$ plasma exposure of the devices prior to the deposition of the PEDOT:PSS solution (Clevios Al 4083 H.C. Stark) is carried out to increase the substrate hydrohilicity.

After baking of the exciton blocking layer $\left(145^{\circ} \mathrm{C}\right.$, $15 \mathrm{~min}$ ) samples are immediately transferred to an organic sublimation vacuum chamber.

Organic material is deposited at controlled deposition parameters (rate: $\sim 0.03 \AA$ $/ \mathrm{s}$ at $p<1 \times 10^{-6}$ mbar). 
$\mathrm{LiF}$ and $\mathrm{Al}$ are deposited using a shadow mask (device active area: $0.125 \mathrm{~cm}^{2}$ ) in a nitrogen filled golvebox compartment and devices are encapsulated using a nitrogen filled sample holder for device testing.

4.4 Electrical device characterization Characterization of devices is carried out using a solar simulator (LOT-Oriel LS0106) at an illumination density of $100 \mathrm{~mW} / \mathrm{cm}^{2}$. A LabView based computer controlled program is used to sweep $I-V$ measurements using a Keithley 2400 sourcemeter.

XRD measurements were conducted on an in-house four circle diffractometer using the $K_{\alpha}$ lines of a molybdenum tube providing X-rays with a mean wavelength of $0.71 \AA$.

Acknowledgements We acknowledge support by the German Excellence Initiative of the Deutsche Forschungsgemeinschaft (DFG) via the "Nanosystems Initiative Munich (NIM)", the German research foundation (DFG) in the program "SPP1355: Elementary processes of organic photovoltaics" and via DFG Ni6324-1, the Center for NanoScience (CeNS) Munich, and the EC Marie-Curie ITN-SUPERIOR (PITN-GA-2009-238177).

\section{References}

[1] J. Wu, W. Pisula, and K. Müllen, Chem. Rev. 107, 718 (2007).

[2] S. Sergeyev, W. Pisula, and Y. H. Geerts, Chem. Soc. Rev. 36, 1902 (2007).

[3] W. Pisula, X. Feng, and K. Müllen, Chem. Mater. 23, 554 (2011).

[4] C. D. Simpson, J. Wu, M. D. Watson, and K. M. llen, J. Mater. Chem. 14, 494 (2004).

[5] A. Craats, J. Warman, and A. Fechtenkötter, Adv. Mater. 11, 1469 (1999).

[6] D. Käfer, A. Bashir, X. Dou, G. Witte, K. Müllen, and C. Wöll, Adv. Mater. 22, 384 (2010).

[7] L. Schmidt-Mende, A. Fechtenkotter, K. Mullen, E. Moons, R. H. Friend, and J. D. MacKenzie, Science 293, 1119 (2001).

[8] H. C. Hesse, J. Weickert, M. Al-Hussein, L. Doessel, X. Feng, K. Muellen, and L. Schmidt-Mende, Sol. Energy Mater. Sol. Cells 94, 560 (2010).

[9] Z. Bao, A. J. Lovinger, and J. Brown, J. Am. Chem. Soc. 120, 207 (1998).

[10] H. Peisert, M. Knupfer, T. Schwieger, G. G. Fuentes, D. Olligs, J. Fink, and T. Schmidt, J. Appl. Phys. 93, 9683 (2003).

[11] S. B. Heidenhain, Y. Sakamoto, T. Suzuki, A. Miura, H. Fujikawa, T. Mori, S. Tokito, and Y. Taga, J. Am. Chem. Soc. 122, $10240(2000)$

[12] Y. Sakamoto, T. Suzuki, A. Miura, H. Fujikawa, S. Tokito, and Y. Taga, J. Am. Chem. Soc. 122, 1832 (2000).

[13] A. Yassar, F. Demanze, and A. Jaafari, Adv. Funct. Mater. 12, 699 (2002).
[14] Y. Kikuzawa, T. Mori, and H. Takeuchi, Org. Lett. 9, 4817 (2007).

[15] S. Entani, T. Kaji, S. Ikeda, T. Mori, Y. Kikuzawa, H. Takeuchi, and K. Saiki, J. Phys. Chem. C 113, 6202 (2009).

[16] T. Mori, Y. Kikuzawa, and H. Takeuchi, Org. Electron. 9, 328 (2008).

[17] Z. E. Ooi, T. L. Tam, R. Y. C. Shin, Z. K. Chen, T. Kietzke, A. Sellinger, M. Baumgarten, K. Mullen, and J. C. deMello, J. Mater. Chem. 18, 4619 (2008).

[18] H. Gommans, T. Aernouts, B. Verreet, P. Heremans, A. S. Medina, C. G. Claessens, and T. Torres, Adv. Funct. Mater. 19, 3435 (2009).

[19] P. Sonar, J. P. Fong Lim, and K. L. Chan, Energy Environ. Sci. 4, 1558 (2011).

[20] M. Scharber, D. Mühlbacher, M. Koppe, P. Denk, C. Waldauf, A. Heeger, and C. Brabec, Adv. Mater. 18, 789 (2006).

[21] H. C. Hesse, J. Weickert, C. Hundschell, X. Feng, K. Müllen, B. Nickel, A. J. Mozer, and L. Schmidt-Mende, Adv. Energy Mater. 1, 861 (2011).

[22] R. R. Lunt, J. B. Benziger, and S. R. Forrest, Adv. Mater. 22, 1233-1236 (2010).

[23] W. Pisula, A. Menon, M. Stepputat, I. Lieberwirth, U. Kolb, A. Tracz, H. Sirringhaus, T. Pakula, and K. Mullen, Adv. Mater. 17, 684 (2005).

[24] R. Goddard, M. W. Haenel, W. C. Herndon, C. Krueger, and M. Zander, J. Am. Chem. Soc. 117, 30 (1995).

[25] D. E. Markov, E. Amsterdam, P. W. M. Blom, A. B. Sieval, and J. C. Hummelen, J. Phys. Chem. A 109, 5266 (2005).

[26] P. Shaw, A. Ruseckas, and I. Samuel, Adv. Mater. 20, 3516 (2008).

[27] G. Dennler, M. C. Scharber, and C. J. Brabec, Adv. Mater. 21, 1323 (2009).

[28] R. Lunt, N. Giebink, A. Belak, J. Benziger, and S. Forrest, J. Appl. Phys. 105, 3711 (2009).

[29] F. Xu and D. Yan, Appl. Phys. Lett. 99, (2011).

[30] G. Dennler, M. C. Scharber, T. Ameri, P. Denk, K. Forberich, C. Waldauf, and C. J. Brabec, Adv. Mater. 20, 579 (2008).

[31] T. Ameri, G. Dennler, C. Lungenschmied, and C. J. Brabec, Energy Environ. Sci. 2, 347 (2009).

[32] J. Peet, J. Kim, N. Coates, W. Ma, D. Moses, A. Heeger, and G. Bazan, Nature Mater. 6, 497 (2007).

[33] D. Mühlbacher, M. Scharber, M. Morana, Z. Zhu, D. Waller, R. Gaudiana, and C. Brabec, Adv. Mater. 18, 2884 (2006).

[34] K. Walzer, B. Maennig, M. Pfeiffer, and K. Leo, Chem. Rev 107, 1233 (2007).

[35] D. Venkataraman, S. Yurt, B. H. Venkatraman, and N. Gavvalapalli, J. Phys. Chem. Lett. 1, 947 (2010).

[36] H. C. Hesse, D. Lebmbke, L. Doessel, X. Feng, K. Muellen, and L. Schmidt-Mende, Nanotechnology 22, 055303 (2011).

[37] F. Yang and S. R. Forrest, ACS Nano 2, 1022 (2008) 